\title{
Management of allergic conjunctivitis: an evaluation of the perceived comfort and therapeutic efficacy of olopatadine $0.2 \%$ and azelastine $0.05 \%$ from two prospective studies
}

\author{
Arthur B Epstein' \\ Peter TVan Hoven ${ }^{2}$ \\ Alan Kaufman ${ }^{3}$ \\ Warner W Carr ${ }^{4}$
}

'North Shore Contact Lens and Vision Consultants, Roslyn Heights, NY, USA; ${ }^{2}$ Primary Eyecare Group PC, Brentwood, TN, USA; ${ }^{3}$ Adult Allergy Clinic and the Division of Allergy and Immunology, Our Lady of Mercy Medical Center, Bronx, NY, USA; ${ }^{4}$ Southern California Research, Mission Viejo, CA, USA

Correspondence: Arthur B Epstein North Shore Contact Lens and Vision Consultants, PC Roslyn Heights, NY II577, USA

Tel + I 5162994540

Fax +I 5162994542

Email artepstein@artepstein.com
Purpose: Results from 2 patient-reported outcome studies of allergic conjunctivitis sufferers who used olopatadine $0.2 \%$ and azelastine $0.05 \%$ are analyzed.

Methods: The PACE (Pataday Allergic Conjunctivitis Evaluation) multi-center, prospective, open-label study examined patient perceptions of olopatadine $0.2 \%$ once daily (qd) and previous twice daily (bid) allergic conjunctivitis medications via questionnaire in allergic conjunctivitis sufferers who had previously used bid medication and then initiated olopatadine. A second conjunctival antigen challenge (CAC) study evaluated comfort of 4 allergic conjunctivitis medications.

Results: Forty-nine patients from the PACE study $(\mathrm{N}=125)$ with prior azelastine use were examined. Significantly more patients rated themselves "very satisfied" with current olopatadine use compared with past azelastine use on drop comfort ( $\mathrm{p}<0.0001)$, speed of relief $(\mathrm{p}=0.0004)$, and overall satisfaction $(70 \%$ vs $16 \%, \mathrm{p}<0.0001)$. Significantly more patients reported olopatadine "very effective" against swelling compared with azelastine (47\% vs $8 \%$, $\mathrm{p}=0.0404)$. In the CAC study $(\mathrm{N}=36)$, data from olopatadine $(\mathrm{n}=8)$, azelastine $(\mathrm{n}=9)$ and placebo $(\mathrm{N}=36)$ groups were reported. Olopatadine was rated significantly more comfortable than azelastine upon instillation $(p=0.0223)$, at 30 seconds $(p=0.0479)$, and at 1 minute after instillation $(\mathrm{p}=0.0240)$.

Conclusion: In the reported studies, olopatadine $0.2 \%$ qd was more comfortable than azelastine $0.05 \%$ and preferred by patients with allergic conjunctivitis by a ratio of $4: 1$.

Keywords: allergic conjunctivitis, azelastine, ocular allergy, olopatadine, patient perceptions

\section{Introduction}

Allergic conjunctivitis is a complex disorder that significantly contributes to the burden of misery and economic impact resulting from environmental allergies, especially in those patients who are either untreated or ineffectively treated. ${ }^{1}$ Allergic conjunctivitis is reported to affect approximately $15 \%$ to $20 \%$ of the US population. ${ }^{2}$ Some suggest that as much as $30 \%$ of the US population is affected by seasonal allergy symptoms with as many as $70 \%$ to $80 \%$ of these people having ocular symptoms. ${ }^{3}$ Recent reports suggest that ocular allergy is both under-diagnosed and under-treated; therefore, the actual global impact may be underrepresented. ${ }^{4}$ Seasonal and perennial allergic rhinitis and conjunctivitis cause disruption in daily activities, which is reflected by diminished quality of life measures. ${ }^{1,5}$ Managing allergy effectively requires adequate relief of symptoms and prevention of future symptoms. With this approach, patients have reported enhanced quality of life. ${ }^{1,6}$

The ocular manifestations of seasonal and perennial allergy occur as a result of mast cell degranulation and subsequent allergic inflammation in sensitized individuals. During 
this cascade, allergens bind and cross-link allergen-specific $\operatorname{IgE}$ antibodies on conjunctival mast cell surfaces, initiating degranulation and the release of histamine. Once released, histamine binds to histamine receptors on the conjunctival surface, causing itching, redness, and swelling. ${ }^{7}$ Recent evidence suggests that histamine-stimulated conjunctival epithelial cells may upregulate the allergic inflammatory cascade. ${ }^{8,9}$ Mast cells additionally release both preformed and synthesized mediators that contribute to the allergic response. ${ }^{10,11}$

The comparatively large surface area of the conjunctiva, a robust vascular supply, and a dense concentration of mast cells make allergic conjunctivitis a particularly vexing form of allergy for affected patients. As such, appropriate diagnosis and effective treatment can have a positive impact on patient's quality of life and disease management. In addition, meeting a patient's perceived needs is paramount for effective allergy management, particularly with ocular allergy, due to the eyes' habitual but necessary exposure to the environment.

The newest class of topical anti-allergy medication for allergic conjunctivitis is the dual-action agent, which combines strong antihistaminic activity (providing rapid relief) with mast-cell stabilizing properties (responsible for prolonged relief). ${ }^{12}$ Five dual-action agents have earned approval from the US Food and Drug Administration (FDA) for the treatment of allergic conjunctivitis: epinastine $0.05 \%$ (Elestat $^{\circledR}$; Allergan, Inc., Irvine, CA, USA), ketotifen $0.025 \%$ (Zaditor $^{\circledR}$; Novartis Ophthalmics, Duluth, GA, USA), azelastine 0.05\% (Optivar ${ }^{\circledR}$; MedPointe Pharmaceuticals, Somerset, NJ, USA), olopatadine $0.1 \%$ (Patanol $^{\circledR}$; Alcon Laboratories, Inc., Fort Worth, TX, USA), and olopatadine 0.2\% (Pataday ${ }^{\mathrm{TM}}$; Alcon Laboratories, Inc., Fort Worth, TX, USA).

Despite similar classification, there is evidence that significant differences exist between the various agents. Specifically, olopatadine $0.1 \%$, the first dual-action agent approved by the FDA, has demonstrated superior efficacy compared to the other dual-action agents in numerous clinical studies. ${ }^{13-15}$ Olopatadine also demonstrates minimal surface activity on mast cell and corneal epithelial cell membranes, which is dose-dependent and potentially clinically relevant. ${ }^{16}$ As the first agent in its class, olopatadine $0.1 \%$ has become the agent by which all other agents in this class are judged.

Olopatadine $0.2 \%$ was recently introduced in an attempt to improve on the qualities of the $0.1 \%$ formulation. It has demonstrated patient acceptance and clinical efficacy comparable or superior to olopatadine $0.1 \% .{ }^{17}$ Like other dual-action agents, olopatadine $0.2 \%$ is approved for the treatment of itching associated with allergic conjunctivitis. ${ }^{18}$ However, it is the only dual-action agent to be indicated for once daily (qd) dosing, ${ }^{18}$ providing a potential advantage over twice daily (bid) medications. ${ }^{19-22}$ Once daily dosing provides increased convenience and possibly even improved patient adherence to treatment. With more convenient qd dosing, it is likely that olopatadine $0.2 \%$ will supplant its lower concentration predecessor. ${ }^{23}$

Olopatadine $0.2 \%$ has demonstrated superior comfort and efficacy compared to the dual-action agent epinastine $0.05 \% .{ }^{24}$ No controlled clinical studies have been published directly comparing olopatadine $0.2 \%$ and azelastine $0.05 \%$; however, olopatadine $0.1 \%$ has demonstrated superior clinical efficacy compared to azelastine $0.05 \%$ in a comparison using the controlled conjunctival antigen challenge (CAC) model. ${ }^{15}$

To examine the relative clinical characteristics of olopatadine $0.2 \%$ and azelastine $0.05 \%$, this report explores the relationship among clinical efficacy, perceived comfort and therapeutic satisfaction of these 2 agents. Specifically, selected results from 2 independent, prospective, patient-reported outcome studies focusing on allergic conjunctivitis management using either or both olopatadine $0.2 \%$ and azelastine $0.05 \%$ are examined to gain insight into this clinically important paradigm..$^{25,26}$

\section{Methods PACE study}

The PACE (Pataday Allergic Conjunctivitis Evaluation) study was a multi-center, prospective, open-label, single-arm study conducted at 10 allergy, ophthalmology, and optometry practices throughout the US during the spring of 2008 that examined adult patients with allergic conjunctivitis. The purpose of this study was to evaluate patient perceptions of olopatadine $0.2 \%$ and previous bid anti-allergy medication (olopatadine $0.1 \%$, azelastine $0.05 \%$, ketotifen $0.025 \%$, or epinastine $0.05 \%$ used within the last 6 months) for the treatment of allergic conjunctivitis. This report presents data only from those patients in the azelastine $0.05 \%$ group.

Patients 18 years or older with active signs and/or symptoms of allergic conjunctivitis (as assessed by the investigator) who had been treated in the last 6 months with a prescription, topical, ocular, anti-allergy, bid medication were included in this study. Exclusion criteria were any serious ocular or other medical condition that could result in a patient's inability to safely complete the study; hypersensitivity or other contraindication to the use of the study medication or its components; known history of recurrent corneal erosion 
syndrome; ocular trauma in either eye within 3 months prior to Visit 1; any ocular surgical intervention within 6 months prior to Visit 1 or anticipation of ocular surgery during the study; presumed or actual ocular infection or history of ocular herpes in either eye; and any significant illness that could be expected to interfere with the study, including autoimmune disease, psoriasis, eczema, rosacea, severe cardiovascular disease, poorly controlled hypertension, poorly controlled diabetes, history of status asthmaticus, or history of moderate to severe allergic asthma.

On Day 1 (Visit 1), patients completed an allergy questionnaire to evaluate their previous bid medication and investigators completed a medical history. Patients were then instructed to begin using qd olopatadine $0.2 \%$. On Day 7 , patients completed a similar allergy questionnaire to evaluate olopatadine $0.2 \%$. Investigators noted any adverse events or changes in concomitant medication at that time.

The questionnaires asked patients to rate their perceptions of efficacy for their anti-allergy medication (previous bid medication was rated at Day 1 and olopatadine $0.2 \%$ at Day 7 ) with respect to ocular itching, redness, tearing, and swelling using the 5 descriptors: very/somewhat effective, undecided, and very/somewhat ineffective. Patients were also asked to rate their satisfaction with their anti-allergy medication with respect to 3 parameters (drop comfort, speed of relief, and overall satisfaction) using the descriptors: very/somewhat satisfied, undecided, and very/somewhat dissatisfied. Withinpatient changes from baseline to follow-up in the global score were tested using paired $t$ test. Between-group comparisons were carried out using Pearson's chi square test, or Fisher's exact test when sample sizes $<30$. A p value of $\leq 0.05$ was considered statistically significant. Statistical analysis was performed in SAS (PC Version 9.1.2, SAS Institute, Cary, NC, USA) by an independent biostatistician. Safety was assessed via collection of all reported adverse events.

\section{Conjunctival antigen challenge study}

This was a single-center, prospective, double-masked, placebo- and contralaterally controlled CAC study of patients with a documented history of allergic conjunctivitis conducted by Ophthalmic Research Associates, Inc. (North Andover, MA, USA) January to February 2007. The purpose of this CAC study was to determine the comfort of qd olopatadine $0.2 \%$ relative to currently available bid antiallergy medications.

Patients 18 years or older must have manifested a positive allergen challenge reaction (ie, itching and redness) in each eye at both Visit 1 (screening visit) and Visit 2 (confirmatory visit), manifested a positive skin test reaction within the past 24 months to the allergen reportedly causing the allergic conjunctivitis, and had a best-corrected $\log$ MAR visual acuity score of 0.60 or better in each eye. Exclusion criteria included any allergy or contraindication to the use of any study medication, active ocular infection, any ocular or medical condition that could affect study parameters, signs or symptoms of allergic conjunctivitis (greater than 1+ redness or any itching) in either eye at the start of any visit, dry eye syndrome requiring daily use of artificial tear substitute, history of ocular surgery within the past 3 months, use of an investigational drug or device within 30 days before Visit 1 or during the study period, and use of any medications (ie, H1-antagonist antihistamines, mast cell stabilizers, corticosteroids) within 72 hours before Visit 1 or anytime during the study that could interfere with the study parameters.

This study followed a standardized CAC protocol. ${ }^{27}$ After identifying and confirming the proper dosage of a known allergen at Visits 1 and 2, patients were randomized by treatment and by eye to receive 1 of 4 study medications (olopatadine $0.2 \%$, olopatadine $0.1 \%$, azelastine $0.05 \%$, or ketotifen $0.025 \%$ ) in 1 eye. All patients received placebo (Tears Naturale II ${ }^{\circledR}$, Alcon Laboratories, Inc., Fort Worth, TX, USA) in the contralateral eye. This report presents data only from those eyes treated with olopatadine $0.2 \%$, azelastine $0.05 \%$, or placebo. At Visit 3 , investigators instilled study medication (anti-allergy medication in one eye and placebo in the other) 5 minutes prior to and 30 minutes after CAC. Patients assessed drop comfort using an 11-point scale $(0=$ very comfortable to $10=$ very uncomfortable $)$ immediately, 30 seconds, 1 minute, and 2 minutes after second instillation. Differences in mean drop comfort scores between treatment groups were assessed using a paired $t$ test at each time point. Safety variables assessed were corrected distance visual acuity, slit lamp biomicroscopy, and all adverse events (reported, elicited, and observed).

\section{Results PACE study}

A total of 125 patients was enrolled in the PACE study. ${ }^{25}$ This report presents data from the 49 patients with a history of using the bid medication azelastine $0.05 \%$. Forty-eight patients $(98 \%)$ completed the questionnaire at both Day 1 and Day 7; one patient was lost to follow-up. The average age of the 49 patients with a history of azelastine $0.05 \%$ use was 56.6 years (range, 26-85; Table 1). Approximately three-quarters of the patients were female; $51 \%$ were white and $24 \%$ were Hispanic. 
Table I Patient demographics $(\mathrm{N}=49)$

\begin{tabular}{ll}
\hline Age (years) & \\
Mean \pm SD & $56.57 \pm 15.62$ \\
Range & $26-85$ \\
Gender (\%) & \\
Male & $26 \%$ \\
Female & $74 \%$ \\
Race (\%) & \\
White & $51 \%$ \\
Black & $12 \%$ \\
Asian & $8 \%$ \\
Hawaiian & $4 \%$ \\
Hispanic & $24 \%$ \\
\hline
\end{tabular}

Note: Age information was not available in I patient; gender information was not available for 2 patients.

Similar percentages of patients rated olopatadine $0.2 \%$ and azelastine $0.05 \%$ as "somewhat effective" for both itching and redness, but more patients reported that olopatadine was "very effective" compared with azelastine $0.05 \%$ ( $46 \%$ vs $20 \%$ for itching, $42 \%$ vs $17 \%$ for redness; Figure 1). While patients reported similar overall favorable results (somewhat to very effective) between the 2 medications for tearing and swelling, more of these patients reported that olopatadine $0.2 \%$ was "very effective" compared with azelastine $0.05 \%$ ( $25 \%$ vs $9 \%$ for tearing, $47 \%$ vs $8 \%$ for swelling; Figure 2). The difference between medications in relief of swelling was statistically significant $(p=0.0404)$; a trend toward superiority for olopatadine was reported for both itching $(\mathrm{p}=0.0691)$ and redness $(\mathrm{p}=0.0715)$.

Approximately 3 to 4 times as many patients rated themselves "very satisfied" with current olopatadine $0.2 \%$ use compared with past azelastine $0.05 \%$ use on 3 different parameters (Figure 3): drop comfort ( $75 \%$ vs $25 \%$, $\mathrm{p}<0.0001)$, speed of relief ( $60 \%$ vs $20 \%, \mathrm{p}=0.0004)$, and overall satisfaction ( $70 \%$ vs $16 \%, \mathrm{p}<0.0001)$.

Among patients who had used azelastine $0.05 \%, 5$ adverse events in 3 patients were reported during olopatadine $0.2 \%$ treatment: adverse taste $(n=2)$, dry eye $(n=1)$, post-nasal drip $(\mathrm{n}=1)$, and dilated pupil $(\mathrm{n}=1)$. All adverse events resolved without treatment.

\section{Conjunctival antigen challenge study}

Thirty-six patients were enrolled in the CAC study; ${ }^{26}$ data from 17 eyes included in the olopatadine $0.2 \%(n=8)$ and azelastine $0.05 \%(\mathrm{n}=9)$ groups and all 36 contralateral eyes included in the placebo groups are reported here. Upon instillation, olopatadine $0.2 \%$ was rated significantly more comfortable than azelastine $0.05 \%$ (mean comfort score, 2.8 vs $7.6, p=0.0223$ ) and was indistinguishable from that of placebo (mean comfort score, 2.8; Figure 4). Patients using olopatadine $0.2 \%$ also reported significantly better comfort scores than patients using azelastine $0.05 \%$ at both 30 seconds

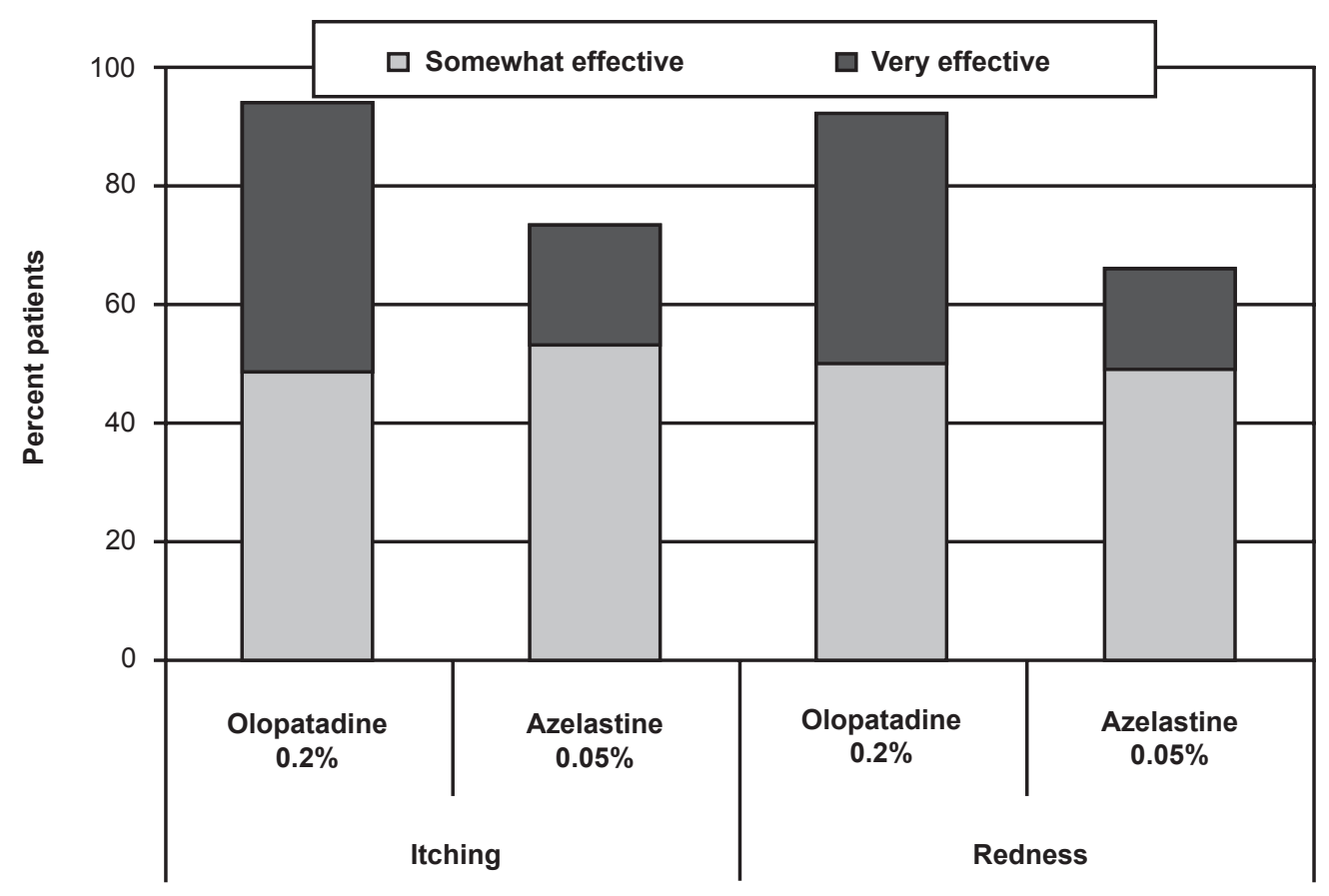

Figure I Patient perceptions of itching and redness (PACE study). 


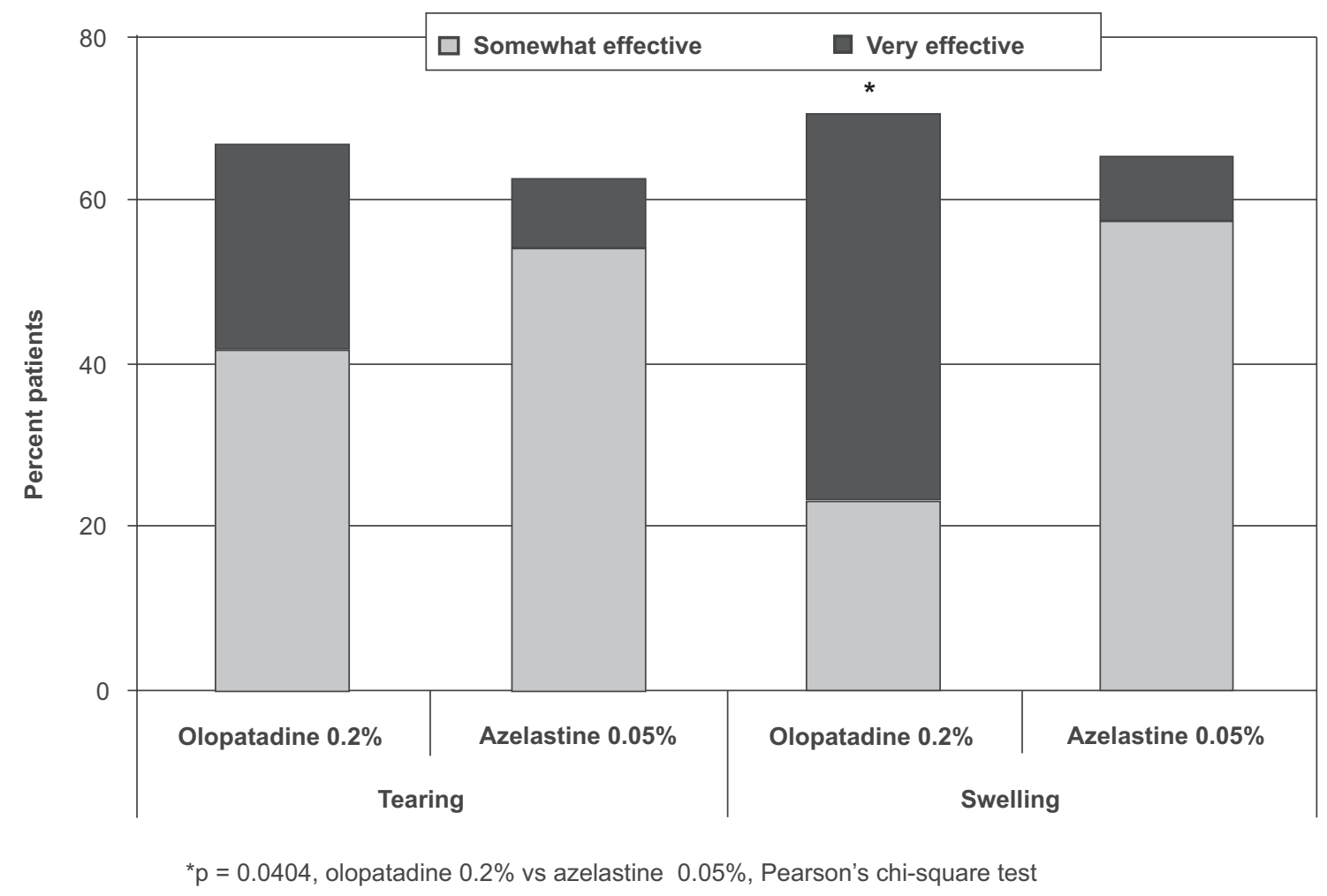

Figure 2 Patient perceptions of tearing and swelling (PACE study).

$(\mathrm{p}=0.0479)$ and 1 minute $(\mathrm{p}=0.0240)$; although olopatadine $0.2 \%$ was numerically better at 2 minutes, this did not reach statistical significance $(p=0.2984)$. Safety measures were not assessed in this CAC study.

\section{Discussion}

Allergy is a well-recognized and very common life disruptor. Ocular allergy is often overlooked but represents a significant component of the burden of allergic disease for many patients. ${ }^{4}$ The eye is an organ critical for survival yet is directly exposed to the environment and offending allergens. As such, managing ocular allergy can pose a significant therapeutic challenge. Previous studies have shown superior targeting and greater effectiveness of topical treatment compared with systemic therapies in managing ocular allergies. ${ }^{28}$ This analysis compares 2 currently available dual-action topical ocular anti-allergy agents in an attempt to better understand what constitutes therapeutic success from the patient's perspective.

Results from these 2 independent studies reinforce the importance of comfort and perceived clinical efficacy in overall patient satisfaction with therapy. Olopatadine $0.2 \%$ demonstrates superior tolerability compared to azelastine $0.05 \%$ in both studies, with significantly greater comfort upon instillation. In addition, patients from the PACE study consistently rated olopatadine $0.2 \%$ more favorably in the efficacy endpoints of itching, redness, tearing, and swelling, although swelling was the only efficacy parameter to reach statistical significance. Patients from this study also reported an increase in overall satisfaction with olopatadine $0.2 \%$. Greater efficacy and tolerability likely play a role in the increased satisfaction with olopatadine $0.2 \%$ of patients with allergic conjunctivitis. ${ }^{29}$

Although olopatadine $0.1 \%$, the original bid formulation, had been previously judged to be significantly more comfortable than azelastine $0.05 \%$ in a randomized, double-masked, crossover study of 91 patients with allergic conjunctivitis, ${ }^{30}$ the increased concentration of olopatadine $0.2 \%$ makes tolerability a valid question. However, the current studies demonstrate that the comfort of this compound relative to azelastine is maintained even at the higher concentration, showing 3 times as many patients who were "very satisfied" with olopatadine's drop comfort compared with azelastine $0.05 \%$ ( $75 \%$ vs $25 \%$, PACE study). These results are supported by results from a patient perception study in which patients who had used both olopatadine $0.1 \%$ and $0.2 \%$ rated them equally comfortable. ${ }^{17}$

The reasons for the superior comfort of olopatadine $0.2 \%$ compared with azelastine $0.05 \%$ have not been clearly defined, but they may be at least partly attributed to $\mathrm{pH}$ 


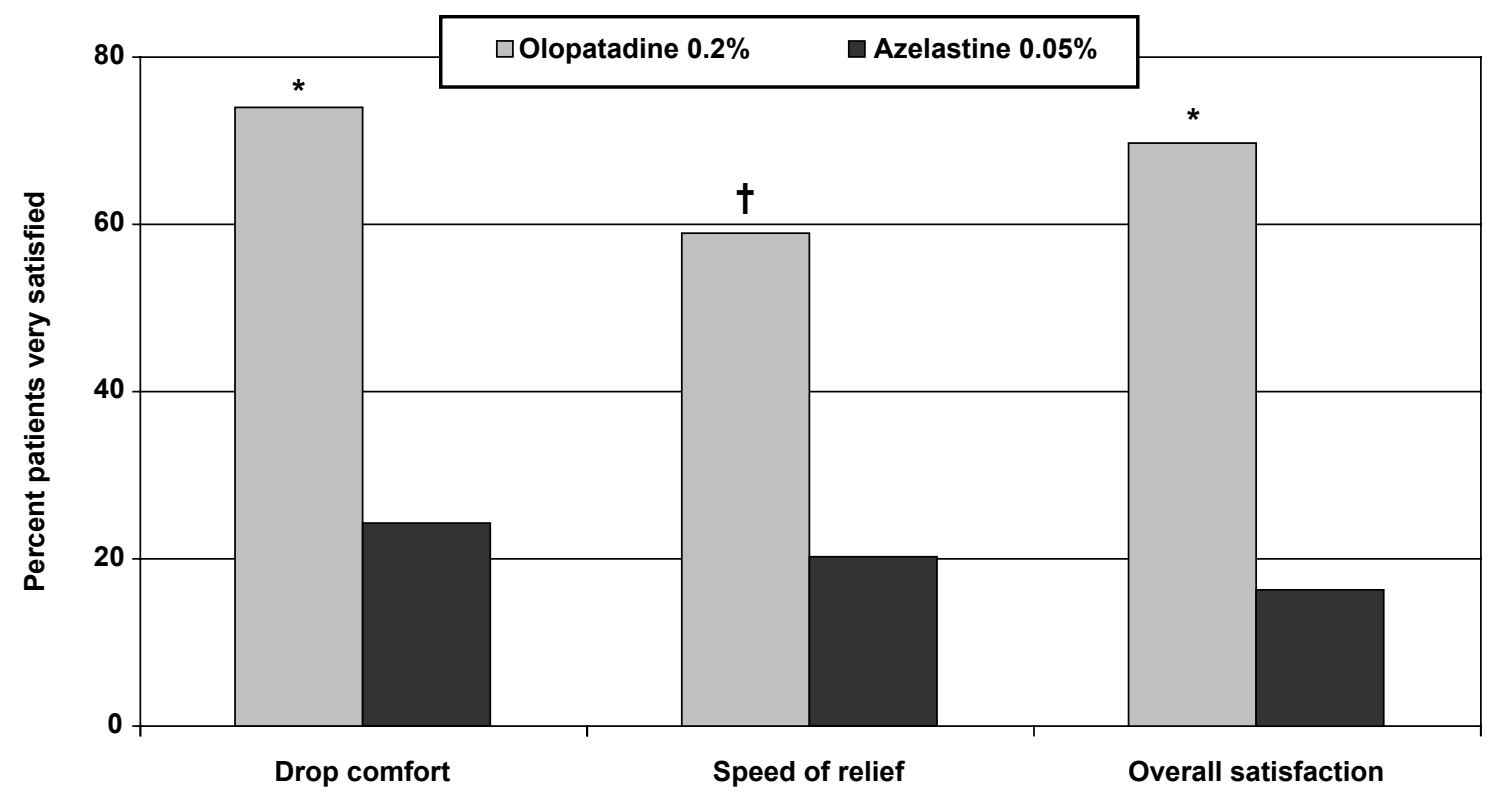

${ }^{*} p<0.0001$, olopatadine $0.2 \%$ vs azelastine $0.05 \%$, Pearson's chi-square test ${ }^{\dagger} \mathrm{p}=0.0004$, olopatadine $0.2 \%$ vs azelastine $0.05 \%$, Pearson's chi-square test

Figure 3 Patient satisfaction (PACE study).

differences; azelastine $0.05 \%$ has a more acidic $\mathrm{pH}$, ranging from 5.0 to $6.5,{ }^{21}$ whereas olopatadine $0.2 \%$ has a physiologic $\mathrm{pH}$ of approximately $7 .{ }^{18}$ Another possible explanation for the greater comfort of olopatadine may arise from the differing effects of these drugs on cell membrane integrity; unlike azelastine and other dual-action agents, olopatadine does not perturb the membranes of ocular surface epithelial cells, the damage of which may explain the stinging and burning that can be encountered upon instillation of dual-action agents. ${ }^{16}$

The patient-reported results from the PACE study, showing a clear advantage of current olopatadine $0.2 \%$ use over past azelastine $0.05 \%$ use for swelling and a numeric trend toward superiority for itching and redness, are consistent with the

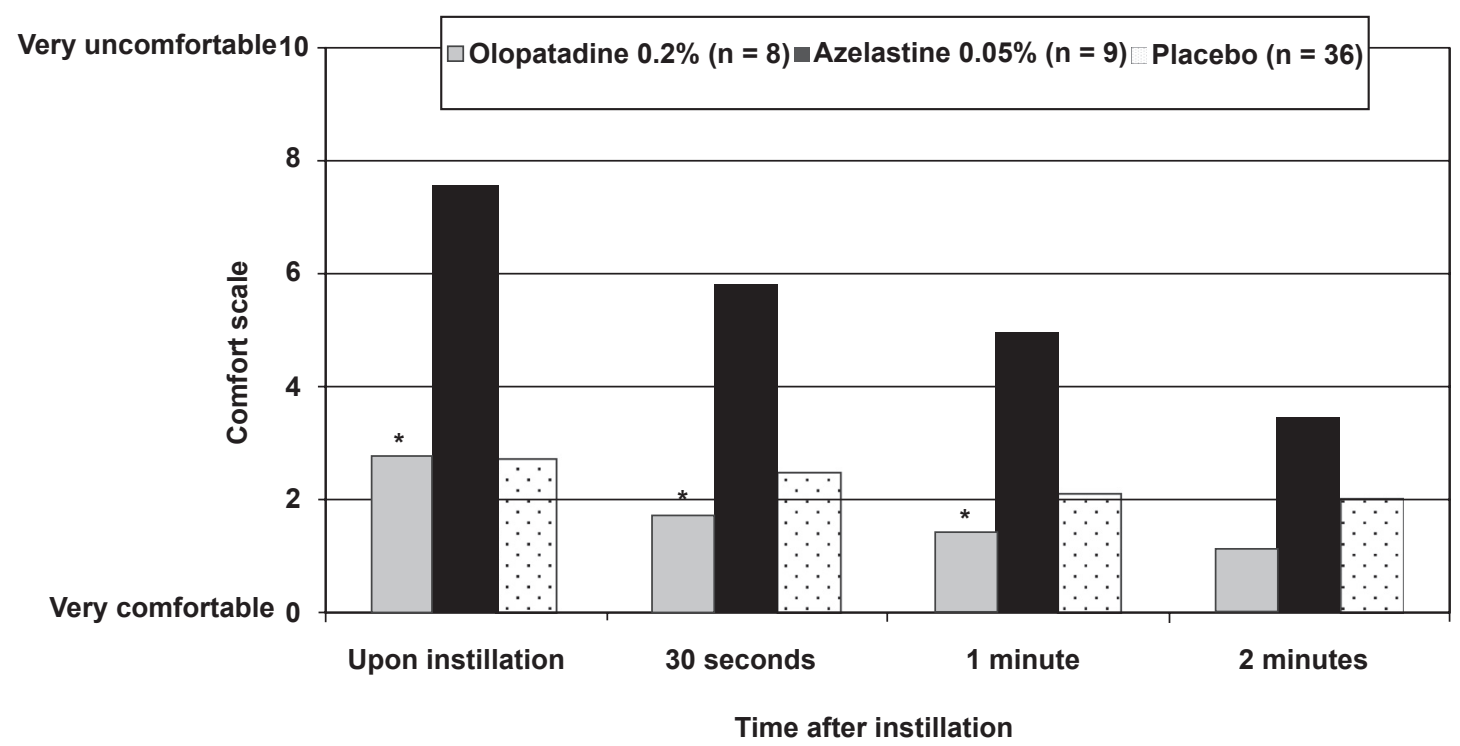

* $p$ value, olopatadine $0.2 \%$ vs azelastine $0.05 \%$; paired $t$ test: upon instillation, $p=0.0223$;

30 seconds, $p=0.0479 ; 1$ minute, $p=0.0240$

Figure 4 Patient drop comfort (CAC study). 
investigator-reported efficacy results from the well-controlled CAC study published by Spangler and colleagues..$^{15}$ This study established the clinical superiority of the $0.1 \%$ formulation of olopatadine over azelastine. Increasing the concentration of olopatadine in the $0.2 \%$ formulation would not be expected to have a negative impact on efficacy.

Both of the studies presented here have limitations. The conclusions from the PACE study are limited by its nonrandomized, open-label, single-arm design. Because patients may have used their bid medications as much as 6 months prior to taking the Day 1 allergy questionnaire, patients had to rely on memory, which may have introduced bias. It is also possible that patients might have expected the "new" medication (olopatadine $0.2 \%$ ) to be superior to the previous medication (azelastine $0.05 \%$ ), despite being provided with no information regarding the efficacy of olopatadine $0.2 \%$. Although the CAC study had a randomized, double-masked, controlled trial design, its small size limits its conclusions as well. These confounding variables should be considered when deciding which medications to use in the clinical setting.

\section{Conclusion}

Allergic conjunctivitis is an under-diagnosed and often suboptimally treated component of allergic disease. Although currently available ophthalmic medications have facilitated the management of this common malady, clinically significant differences among these agents have been reported in the literature. ${ }^{13-15}$ Effective therapy requires in-depth understanding of patient perceptions and responses to these therapeutic agents.

This exploration of data from 2 independent studies provides insight into the therapeutic dynamic for successfully managing ocular allergy by comparing 2 agents of the same class that have different clinical performance and patient-perceived qualities. Among the patients in these 2 studies, olopatadine $0.2 \%$ dosed once daily proved more comfortable and was perceived as clinically more efficacious than azelastine $0.05 \%$. Patient perception is critically important in ensuring sustained compliance and overall satisfaction with both the treatment and the provider.

\section{Acknowledgments}

The authors thank Jennifer Klem, $\mathrm{PhD}$, for medical writing contributions.

\section{Disclosures}

Funding for the studies was provided by Alcon Laboratories, Inc.

\section{References}

1. Scoper SV, Berdy GJ, Lichtenstein SJ, et al. Perception and quality of life associated with the use of olopatadine $0.2 \%$ (Pataday) in patients with active allergic conjunctivitis. Adv Ther. 2007;24(6): $1221-1232$.

2. Ciprandi G, Buscaglia S, Cerqueti PM, Canonica GW. Drug treatment of allergic conjunctivitis. A review of the evidence. Drugs. 1992;43(2):154-176.

3. Katelaris $\mathrm{CH}$, Bielory L. Evidence-based study design in ocular allergy trials. Curr Opin Allergy Clin Immunol. 2008;8(5):484-488.

4. Bielory L. Ocular allergy overview. Immunol Allergy Clin North Am. 2008;28(1):1-23, v.

5. Schatz M. A survey of the burden of allergic rhinitis in the USA. Allergy. 2007;62 (Suppl 85):9-16.

6. Berger W, Abelson MB, Gomes PJ, et al. Effects of adjuvant therapy with $0.1 \%$ olopatadine hydrochloride ophthalmic solution on quality of life in patients with allergic rhinitis using systemic or nasal therapy. Ann Allergy Asthma Immunol. 2005;95(4):361-371.

7. Bielory L, Ghafoor S. Histamine receptors and the conjunctiva. Curr Opin Allergy Clin Immunol. 2005;5(5):437-440.

8. Sharif NA, Xu SX, Yanni JM. Olopatadine (AL-4943A): ligand binding and functional studies on a novel, long acting H1-selective histamine antagonist and anti-allergic agent for use in allergic conjunctivitis. J Ocul Pharmacol Ther. 1996;12(4):401-407.

9. Galatowicz G, Ajayi Y, Stern ME, Calder VL. Ocular anti-allergic compounds selectively inhibit human mast cell cytokines in vitro and conjunctival cell infiltration in vivo. Clin Exp Allergy. 2007;37(11):1648-1656.

10. Bielory L. Allergic and immunologic disorders of the eye. Part II: ocular allergy. J Allergy Clin Immunol. 2000;106(6):1019-1032.

11. Church MK, Holgate ST, Shute JK, et al. Mast cell derived mediators. In: Middleton E, Reed CE, Ellis ET, et al. editors. Allergy Principles and Practice. St. Louis, MO: Mosby;1988. p. 146-167.

12. Leonardi A. New drug treatments for ocular allergies. Expert Rev Ophthalmol. 2007;(2):397-408.

13. Berdy GJ, Spangler DL, Bensch G, Berdy SS, Brusatti RC. A comparison of the relative efficacy and clinical performance of olopatadine hydrochloride $0.1 \%$ ophthalmic solution and ketotifen fumarate $0.025 \%$ ophthalmic solution in the conjunctival antigen challenge model. Clin Ther. 2000;22(7):826-33.

14. Lanier BQ, Finegold I, D'Arienzo P, Granet D, Epstein AB, Ledgerwood GL. Clinical efficacy of olopatadine vs epinastine ophthalmic solution in the conjunctival allergen challenge model. Curr Med Res Opin. 2004;20(8):1227-1233.

15. Spangler DL, Bensch G, Berdy GJ. Evaluation of the efficacy of olopatadine hydrochloride $0.1 \%$ ophthalmic solution and azelastine hydrochloride $0.05 \%$ ophthalmic solution in the conjunctival allergen challenge model. Clin Ther. 2001;23(8):1272-1280.

16. Brockman HL, Momsen MM, Knudtson JR, Miller ST, Graff G, Yanni JM. Interactions of olopatadine and selected antihistamines with model and natural membranes. Ocul Immunol Inflamm. 2003;11(4):247-268.

17. Abelson MB, Spangler DL, Epstein AB, et al. Efficacy of once-daily olopatadine $0.2 \%$ ophthalmic solution compared to twice-daily olopatadine $0.1 \%$ ophthalmic solution for the treatment of ocular itching induced by conjunctival allergen challenge. Curr Eye Res. 2007;32(12):1017-1022.

18. Pataday ${ }^{\mathrm{TM}}$ [package insert]. Fort Worth, TX: Alcon Laboratories, Inc; 2006.

19. Elestat ${ }^{\mathbb{}}$ [package insert]. Irvine, CA: Allergan, Inc; 2003.

20. Patanol ${ }^{\circledR}$ [package insert]. Fort Worth, TX: Alcon Laboratories, Inc; 2003.

21. Optivar ${ }^{\circledR}$ [package insert]. Somerset, NJ: MedPointe Pharmaceuticals; 2003.

22. Zaditor ${ }^{\circledR}$ [package insert]. Duluth, GA: Novartis Ophthalmics; 2001. 
23. Abelson MB, Gomes PJ. Olopatadine $0.2 \%$ ophthalmic solution: the first ophthalmic antiallergy agent with once-daily dosing. Expert Opin Drug Metab Toxicol. 2008;4(4):453-461.

24. Mah FS, Rosenwasser LJ, Townsend WD, Greiner JV, Bensch G. Efficacy and comfort of olopatadine $0.2 \%$ versus epinastine $0.05 \%$ ophthalmic solution for treating itching and redness induced by conjunctival allergen challenge. Curr Med Res Opin. 2007;23(6):1445-1452.

25. Chipps BE, Gower R, Kaufman A, et al. A multi-center study evaluating patient perceptions of olopatadine $0.2 \%$ in patients with allergic conjunctivitis [abstract]. 2008 Western Society of Asthma, Allergy, and Immunology annual meeting.

26. Carr WW. Comparison of drop comfort of four marketed ocular anti-allergic medications [abstract]. 2007 Eastern Allergy Conference annual meeting.

27. Abelson MB, Schaefer K. Conjunctivitis of allergic origin: immunologic mechanisms and current approaches to therapy. Surv Ophthalmol. 1993;38 (Suppl):115-132.
28. Abelson MB, Welch DL. An evaluation of onset and duration of action of patanol (olopatadine hydrochloride ophthalmic solution $0.1 \%$ ) compared to Claritin (loratadine $10 \mathrm{mg}$ ) tablets in acute allergic conjunctivitis in the conjunctival allergen challenge model. Acta Ophthalmol Scand Suppl. 2000;(230):60-63.

29. Scoper SV, Berdy GJ, Lichtenstein SJ, et al. Perception and quality of life associated with the use of olopatadine $0.2 \%$ (Pataday) in patients with active allergic conjunctivitis. Adv Ther. 2007;24(6): 1221-1232.

30. D'Arienzo PA, Granet DB. Variability of drop comfort and its importance as a criterion in the selection of topical therapy for ocular allergy [abstract]. 2001 American Academy of Allergy, Asthma, and Immunology annual meeting, abstract \#2002047. 